\title{
A CONSERVATIVE DISCONTINUOUS GALERKIN METHOD FOR THE DEGASPERIS-PROCESI EQUATION*
}

\author{
HAILIANG LIU $^{\dagger}$, YUNQING HUANG ${ }^{\ddagger}$, AND NIANYU YI
}

To Stanley Osher on his 70th birthday with friendship and appreciation

\begin{abstract}
In this work, we design, analyze and test a conservative discontinuous Galerkin method for solving the Degasperis-Procesi equation. This model is integrable and admits possibly discontinuous solutions, and therefore suitable for modeling both short wave breaking and long wave propagation phenomena. The proposed numerical method is high order accurate, and preserves two invariants, mass and energy, of this nonlinear equation, hence producing wave solutions with satisfying long time behavior. The $L^{2}$-stability of the scheme for general solutions is a consequence of the energy preserving property. The numerical simulation results for different types of solutions of the nonlinear Degasperis-Procesi equation are provided to illustrate the accuracy and capability of the method.
\end{abstract} bility.

Key words. Discontinuous Galerkin method, Degasperis-Procesi equation, conservation, sta-

AMS subject classifications. 65M60, 65M12, 35Q53.

1. Introduction. In this paper, we are interested in numerical approximations to the Degasperis-Procesi (DP) equation:

$$
u_{t}-u_{x x t}=u u_{x x x}+3 u_{x} u_{x x}-4 u u_{x}, \quad x \in \mathbb{R}, t>0,
$$

where the subscript $t$ (or $x$, respectively) denotes the differentiation with respect to time variable $t$ (or $x$ ). We develop a conservative discontinuous Galerkin method (DDG) for this nonlinear DP equation. Our proposed scheme is high order accurate, and preserves two invariants, momentum and energy, of the nonlinear equation, hence producing wave solutions with long time satisfying behavior. The $L^{2}$-stability of the scheme for general solutions is a consequence of the energy preserving property.

The DP equation was found in a study by Degasperis and Procesi [19], as one of three equations in the family

$$
u_{t}-\alpha^{2} u_{x x t}+\gamma u_{x x x}+c_{0} u_{x}=\left(c_{1} u^{2}+c^{2} u_{x}^{2}+c_{3} u u_{x x}\right)_{x},
$$

satisfying "asymptotic integrability to third order", a necessary condition for complete integrability. The other two cases are the Korteweg-de Vries (KdV) equation,

$$
u_{t}+u u_{x}+u_{x x x}=0
$$

and the Camassa-Holm $(\mathrm{CH})$ shallow water equation,

$$
u_{t}-u_{x x t}=u u_{x x x}+2 u_{x} u_{x x}-3 u u_{x} .
$$

Both $\mathrm{CH}$ equation and DP equation are also found in the $\theta$ - class of dispersive models:

$$
u_{t}-u_{t x x}+u u_{x}=\theta u u_{x x x}+(1-\theta) u_{x} u_{x x},
$$

\footnotetext{
${ }^{*}$ Received January 26, 2013; accepted for publication October 2, 2013.

${ }^{\dagger}$ Iowa State University, Mathematics Department, Ames, IA 50011, USA (hliu@iastate.edu).

${ }_{\ddagger}^{\ddagger}$ Hunan Key Laboratory for Computation and Simulation in Science and Engineering; School of Mathematics and Computational Science, Xiangtan University, Xiangtan 411105, P. R. China (huangyq@xtu.edu.cn; yinianyu365109@126.com).
} 
when taking $\theta=1 / 3$ and $\theta=1 / 4$, respectively. The $\theta$-class was identified by Liu in [36], and its analysis on global existence vs. finite wave breaking was given by Liu and Yin [37] with the following results: for $\frac{1}{2} \leq \theta \leq 1$, initial smoothness will persist for all time; if $0 \leq \theta<\frac{1}{2}$, strong solutions of the $\theta$-equation may loss certain regularity in finite time, and traveling waves such as periodic, solitary, peakon, peaked periodic, cusped periodic, or cusped soliton are all permissible [31, 26].

The two special equations, the $\mathrm{CH}$ equation and the DP equation, in the $\theta$-class share several interesting properties including the bi-Hamiltonian structure, the complete integrability, and the soliton-like peakon solutions, and both can be viewed as models of shallow water waves $[23,3,4,29,18,28,12]$. However, there are also important differences: from the perspective of PDE theory, the solutions of the $\mathrm{CH}$ equation basically belong to $H^{1}(\mathbb{R})$ (the first order Sobolev space), while the DP equation can develop shocks (jump discontinuities) which may be understood as entropy solutions $[7,8,38]$.

Furthermore there are differences in invariants. The $\mathrm{CH}$ equation has the following three invariants:

$$
E_{1}=\int_{\mathbb{R}}\left(u-u_{x x}\right) d x, \quad E_{2}=\int_{\mathbb{R}}\left(u^{2}+u_{x}^{2}\right) d x, \quad E_{3}=\int_{\mathbb{R}}\left(u^{3}+u u_{x}^{2}\right) d x .
$$

For the DP equation, the first three invariants are

$$
E_{1}=\int_{\mathbb{R}}\left(u-u_{x x}\right) d x, \quad E_{2}=\int_{\mathbb{R}}\left(u-u_{x x}\right) v d x, \quad E_{3}=\int_{\mathbb{R}} u^{3} d x
$$

where $v$ is defined as

$$
4 v-v_{x x}=u
$$

As can be easily seen, the invariants are substantially different, and there is no simple transformation that casts the DP cases into the $\mathrm{CH}$ cases and vice versa. Solutions in the DP equation have more complicated structure than those in the $\mathrm{CH}$ equation. Particularly, when solutions of the DP equation are discontinuous, one has to impose proper entropy condition to single out the physically relevant solution. These together introduce new difficulties in numerical computations. It is a challenge to design stable and high order accurate numerical schemes for solving the DP equation.

In recent years, a lot of analysis has been given to the DP equation. Coclite and Karlsen [7] showed that the DP equation could admit discontinuous (shock wave) solutions and proved a well-posedness theory in functional spaces containing discontinuous functions. Yin studied the global well-posedness of the DP equation within certain functional classes in a series of papers $[54,55,56]$. Some blow-up results were obtained in $[20,21,36]$. Explicit or special solutions were also studied, for example, entropy shock solutions [38], the general $n$-peakon solutions [40], multi-soliton solutions [41, 42], and traveling wave solutions [31, 26].

Despite of difficulties in dealing with the DP equation, several numerical studies have appeared in the literature. Operator splitting schemes were proposed for the DP equation in $[9,22]$. In [27], a particle method based on the multi-shock-peakon solutions was proposed to investigate entropy weak solutions. These numerical methods were mainly intended to capture shock solutions. Recently, Xu and Shu developed two local discontinuous Galerkin (LDG) methods for the DP equation in [47], with provable stability property and excellent numerical results. 
On the other hand, both $\mathrm{CH}$ equation and DP equation are integrable systems, numerical methods preserving their invariants are advantageous: besides the high accuracy of numerical solutions, an invariant preserving scheme can preserve good stability properties after long-time numerical integration. Much more effort has been devoted in this topic for different integrable PDEs recently [24, 5, 44, 43]. For the DP equation Miyatake and Matsuo [45] proposed two conservative finite difference schemes based on the bi-Hamiltonian structure, both schemes conserving $E_{1}$ and $E_{3}$.

The goal of this paper is to develop a discontinuous Galerkin (DG) method to preserve both momentum and energy at the discrete setting. The DG method is a class of finite element methods using completely discontinuous piecewise-polynomials for the numerical solution and the test functions. It was first designed and has been successful for solving first order PDEs such as nonlinear conservation laws [46, 14, 13, $15,16]$. The local discontinuous Galerkin (LDG) method is an extension of the DG method for solving higher order PDEs. It was first designed for convection-diffuion equations [17], and has been extended to other higher order wave equations, including the $\mathrm{KdV}$ equation [52, 48, 33, 49] and the $\mathrm{CH}$ equation [50], see also the recent review paper [51] on the LDG methods for higher order PDEs. The idea of the LDG method is to rewrite higher order equations into a first order system, and then apply the DG method on the system. In contrast, the direct discontinuous Galerkin (DDG) methods, proposed in $[34,35]$ primarily for diffusion equations, aimed at directly solving higher order PDEs by the DG discretization. There are other recent works sharing the direct feature, such as those by Van Leer and Nomura in [30], Gassner et al. in [25], and Cheng and Shu in [2], all based on certain weak formulation derived from repeated integration by parts for the diffusion term. One main advantage of such methods is the use of special numerical fluxes such that the numerical scheme is not only highly accurate but also satisfies further solution properties, without rewriting the equation into a first order system, see e.g., $[1,53]$ for energy preserving DG methods for KdV type equations.

In this work we propose a novel DG method based on formulation

$$
u_{t}+f(u)_{x}+\psi=0, \quad \psi-\psi_{x x}=3 f(u)_{x} .
$$

This formulation was used in [47], in which the second equation was rewritten into a first order system for applying the LDG discretization. Their methods use dissipative numerical fluxes for the nonlinear advection. Our strategy is different in two aspects: (i) we apply the DDG discretization to solve $\psi$ from the second equation in (1) directly, and then solve $u$ by the DG method applied to the first equation in (1); (ii) the choice of our numerical fluxes is made so that the resulting scheme preserves two integrals $E_{1}$ and $E_{2}$ in smooth region. In the region with strong shocks we adopt an adaptive flux for the nonlinear advection, leading to certain decay of $E_{2}$. From now on, we shall name the method developed in this work as DDG, with $E_{2}$ being conservative or dissipative. Our method gives the desired $k+1$ order of accuracy for polynomial elements of degree $k$. The main feature of the scheme is its capability to produce wave solutions with satisfying long time behavior. Some comparison of long time simulation vividly shows that the scheme which conserves two integrals has much smaller error in phase than those produced by the scheme with a dissipative numerical flux for the quadratic advection.

Outline of the paper. Our purpose here is to present some new DG methods to compute the DP solutions, which are even less regular than peakons and provide numerical evidence of what happens after a peakon- antipeakon collision. In section 2, 
we formulate our DDG method with an adaptive flux, that is wherever strong shocks may appear we use a dissipative flux for the nonlinear convection, elsewhere we use the conservative flux. The DDG method is proved to be energy preserving, and $L^{2}$ stable. In section 3, we present various numerical examples to illustrate the capacity of the DDG scheme to capture various peakons and their interactions.

\section{The discontinuous Galerkin methods.}

2.1. Reformulation. The sense that peakons are weak solutions can be specified by rewriting the DP equation as

$$
0=u_{t}-u_{t x x}+4 f(u)_{x}-f(u)_{x x x}=\left(1-\partial_{x}^{2}\right)\left[u_{t}+f(u)_{x}\right]+3 f(u)_{x},
$$

where $f(u)=u^{2} / 2$, and then apply the formal inverse of $1-\partial_{x}^{2}$, which is $\left(1-\partial_{x}^{2}\right)^{-1} f=$ $\frac{1}{2} G * f=\frac{1}{2} \int_{R} e^{-|y|} f(x-y) d y$. This gives another form of the DP equation,

$$
u_{t}+\partial_{x}[f(u)+3 G * f(u)]=0,
$$

which is formally equivalent to the system

$$
\left\{\begin{aligned}
u_{t}+f(u)_{x}+\psi & =0 \\
\psi-\psi_{x x}-3 f(u)_{x} & =0 .
\end{aligned}\right.
$$

Weak solutions (not only peakons) are then defined as functions that satisfy this conservation law (with nonlocal flux term) in the usual distributional sense. Further in [8] Coclite and Karlsen defined an entropy weak solution of the DP equation as a weak solution which in addition belongs to $L^{\infty}(0, T ; B V(R))$ for all $T>0$ and satisfies the Kruzkov-type entropy condition

$$
\partial_{t} \eta(u)+\partial_{x} q(u)+3 \eta^{\prime}(u) \partial_{x} G * f(u) \leq 0,
$$

in the distributional sense, for all convex $C^{2}$ entropies $\eta$ with the corresponding entropy flux $q$ defined by $q^{\prime}(u)=\eta^{\prime}(u) u$. Then they showed that for initial data $u_{0} \in L^{1}(R) \cap B V(R)$ there exists a unique entropy weak solution of the DP equation. Coclite, Karlsen, and Risebro [9] have designed numerical methods for computing such entropy weak solutions of the DP equation.

Three useful conservation laws for the DP equation are

$$
E_{1}(u)=\int_{\mathbb{R}} u d x, \quad E_{2}(u)=\int_{\mathbb{R}}\left(u-u_{x x}\right) v d x, \quad E_{3}(u)=\int_{\mathbb{R}} u^{3} d x,
$$

where

$$
4 v-v_{x x}=u
$$

Here and in what follows we use the total mass $\int_{\mathbb{R}} u d x$ which equals to the total momentum $\int_{\mathbb{R}}\left(u-u_{x x}\right) d x$ at continuous level. Also $E_{2}$ may be rewritten through integration by parts and using $v_{x x}=4 v-u$,

$$
E_{2}=\int_{\mathbb{R}}\left(u v-u v_{x x}\right) d x=\int_{\mathbb{R}}\left(u^{2}-3 u v\right) d x .
$$

Using (4) and integration by parts again, we may express $E_{2}$ in terms of $v$ as

$$
E_{2}=\int_{\mathbb{R}}\left(4 v^{2}+5\left(v_{x}\right)^{2}+\left(v_{x x}\right)^{2}\right) d x
$$

which has a definite sign. 
2.2. DDG formulation. We develop a direct discontinuous Galerkin (DDG) method for the DP equation subject to initial data $u_{0}(x)$ and periodic boundary conditions. Let us denote the computational mesh of the domain $I$ by $I_{j}=\left(x_{j-1 / 2}, x_{j+1 / 2}\right)$ for $j=1, \ldots, N$. The center of the cell is $x_{j}=\left(x_{j-1 / 2}+x_{j+1 / 2}\right) / 2$, and $h_{j}=$ $x_{j+1 / 2}-x_{j-1 / 2}$. We denote by $w_{j+1 / 2}^{+}$the value of $w$ at $x_{j+1 / 2}$ evaluated from the right element $I_{j+1}$, and $w_{j+1 / 2}^{-}$the value of $w$ at $x_{j+1 / 2}$ evaluated from the left element $I_{j} .[w]=w^{+}-w^{-}$denotes the jump of $w$ at cell interfaces, and $\{w\}=\frac{1}{2}\left(w^{+}+w^{-}\right)$ denotes the average of the left and right interface values. We then define the piecewise polynomial space $V_{h}$ as the space of polynomials of degree $k$ in each cell $I_{j}$, i.e.,

$$
V_{h}=\left\{w: w \in P^{k}\left(I_{j}\right) \text { for } x \in I_{j}, j=1, \ldots, N\right\} .
$$

For any element $I_{j}, j=1, \ldots, N$, we define the inner product and $L^{2}$ norm in $I_{j}$ as

$$
(w, r)_{I_{j}}=\int_{I_{j}} w r d x, \quad\|w\|_{I_{j}}^{2}=(w, w)_{I_{j}},
$$

for the functions $w, r$.

The DDG method is formulated as follows: find $u_{h}, \psi_{h} \in V_{h}$, such that $\forall w, r \in V_{h}$,

$$
\begin{array}{r}
\left(u_{h t}, w\right)_{I_{j}}-\left(f\left(u_{h}\right), w_{x}\right)_{I_{j}}+\left.\hat{f}\left(u_{h}\right) w\right|_{\partial I_{j}}+\left(\psi_{h}, w\right)_{I_{j}}=0 \\
\left(\psi_{h}, r\right)_{I_{j}}+\left(\psi_{h x}, r_{x}\right)_{I_{j}}-\left.\hat{\psi}_{h x} r\right|_{\partial I_{j}}-\left.\left(\psi_{h}-\hat{\psi}_{h}\right) r_{x}\right|_{\partial I_{j}} \\
+3\left(f\left(u_{h}\right), r_{x}\right)_{I_{j}}-\left.3 \hat{f}\left(u_{h}\right) r\right|_{\partial I_{j}}=0
\end{array}
$$

with the numerical fluxes

$$
\hat{f}\left(u_{h}\right)=\frac{\int_{u_{h}^{-}}^{u_{h}^{+}} f(u) d u}{\left[u_{h}\right]}
$$

and

$$
\hat{\psi}_{h x}=\beta \frac{\left[\psi_{h}\right]}{h}+\psi_{h x}^{\theta}, \quad \hat{\psi}_{h}=\psi_{h}^{1-\theta},
$$

where

$$
\psi_{h x}^{\theta}:=\theta \psi_{h x}^{+}+(1-\theta) \psi_{h x}^{-}, \quad \psi_{h}^{1-\theta}:=(1-\theta) \psi_{h}^{+}+\theta \psi_{h}^{-} .
$$

Here $\theta \in[0,1]$, and $\beta \geq 1$ is a parameter to be determined.

As for the numerical fluxes, some remarks are in order.

REMARK 2.1. The form of the flux (10) with $\theta=1 / 2$ corresponds to the numerical flux by Liu and Yan in [35] with $\left(\beta_{0}, \beta_{1}\right)=(\beta, 0)$, and that with $\theta=1$ or $\theta=0$ is more related to Cheng and Shu's work [2] using alternative numerical flux for the solution and its derivative. The interesting fact is that for any $\theta \in[0,1]$ in $(10)$ and the flux (9), both $E_{1}$ and $E_{2}$ are preserved at the semi-discrete level for the whole system (2).

REMARK 2.2. The corresponding DDG scheme is called the DDG-C scheme. For discontinuous solutions an entropy flux for $\hat{f}\left(u_{h}\right)$ needs to be used to capture the entropy solution. For example, we may adopt the Lax-Friedrich numerical flux

$$
\hat{f}\left(u_{h}^{-}, u_{h}^{+}\right)=\frac{1}{2}\left(f\left(u_{h}^{-}\right)+f\left(u_{h}^{+}\right)-\sigma\left(u_{h}^{+}-u_{h}^{-}\right)\right),
$$


where $\sigma=\max \left|f^{\prime}(u)\right|$, the resulting DDG scheme is called the DDG-D scheme. In our numerical simulation, we use the local Lax-Friedrich flux with $\sigma=$ $\max _{u \in\left(u_{h}^{-}, u_{h}^{+}\right)}\left|f^{\prime}(u)\right|$.

REMARK 2.3. In our numerical simulation, we adopt an adaptive numerical flux for $\hat{f}\left(u_{h}\right)$,

$$
\hat{f}\left(u_{h}^{-}, u_{h}^{+}\right)= \begin{cases}\frac{\int_{u_{h}^{-}}^{u_{h}^{+}} f(u) d u}{\left[u_{h}\right]}, & \text { if }\left|u_{h}^{+}-u_{h}^{-}\right| \leq 10^{-2}, \\ \frac{1}{2}\left(f\left(u_{h}^{-}\right)+f\left(u_{h}^{+}\right)-\sigma\left(u_{h}^{+}-u_{h}^{-}\right)\right), & \text {otherwise, }\end{cases}
$$

where $\sigma=\max _{u \in\left(u_{h}^{-}, u_{h}^{+}\right)}\left|f^{\prime}(u)\right|$, and $10^{-2}$ may vary as long as it can serve as a shock detector.

REMARK 2.4. For $k=0$, it is necessary to take $\beta=1$ to obtain the following finite volume scheme

$$
\begin{aligned}
& \frac{d}{d t} u_{j}+\frac{\hat{f}_{j+1 / 2}-\hat{f}_{j-1 / 2}}{h}+\psi_{j}=0, \\
& \psi_{j}-\frac{\psi_{j+1}-2 \psi_{j}+\psi_{j-1}}{h^{2}}-3 \frac{\hat{f}_{j+1 / 2}-\hat{f}_{j-1 / 2}}{h}=0,
\end{aligned}
$$

where $\hat{f}$ is defined as above, (7) or (13), but with $u^{+}=u_{j+1}$ and $u^{-}=u_{j}$ at $x_{j+1 / 2}$. Accuracy test of order one is also observed numerically, see Table 1 .

The global form of the DDG formulation may be obtained by summing over all j's,

$$
\begin{aligned}
\left(u_{h t}, w\right)+\mathcal{B}\left(u_{h}, w\right)+\left(\psi_{h}, w\right) & =0, \quad \forall w \in V_{h}, \\
\left(\psi_{h}, r\right)+\mathcal{D}\left(\psi_{h}, r\right)+3 \mathcal{B}\left(u_{h}, r\right) & =0, \quad \forall r \in V_{h},
\end{aligned}
$$

where the two operators are defined by

$$
\mathcal{B}(w, r):=-\sum_{j=1}^{N}\left(f(w), r_{x}\right)_{I_{j}}-\sum_{j=1}^{N}(\hat{f}(w)[r])_{j-\frac{1}{2}}
$$

and

$$
\mathcal{D}(w, r):=\sum_{j=1}^{N}\left(w_{x}, r_{x}\right)_{I_{j}}+\sum_{j=1}^{N}\left(\hat{w}_{x}[r]+\left[w r_{x}\right]-\hat{w}\left[r_{x}\right]\right)_{j-\frac{1}{2}}
$$

We can rewrite the operator $\mathcal{B}$ defined by (16) as

$$
\mathcal{B}(w, w)=\sum_{j=1}^{N}\left(\alpha\left(\hat{f}, u^{*}\right)[u]^{2}\right)_{j-1 / 2}
$$

where $\alpha\left(\hat{f}, u^{*}\right)=[u]^{-1}\left(f\left(u^{*}\right)-\hat{f}\right)$ with $f\left(u^{*}\right)=[u]^{-1} \int_{u^{-}}^{u^{+}} f(\xi) d \xi$.

With the choice of $\hat{f}$ in (9), we have $\hat{f}=f\left(u^{*}\right)$, hence $\alpha\left(\hat{f}, u^{*}\right)=0$, then the scheme is conservative in the sense that

$$
\mathcal{B}(w, w)=0, \quad \forall w \in V_{h} .
$$


For the monotone flux, $\hat{f}(\uparrow, \downarrow)$, including the Lax-Friedrich flux (12), it is known that $\alpha \geq 0$, see e.g., $[2,32]$, then the scheme is dissipative in the sense that

$$
\mathcal{B}(w, w) \geq 0, \quad \forall w \in V_{h} .
$$

The operator $\mathcal{D}$ defined by (17) with the choice of $\hat{w}_{x}$ and $\hat{w}$ in $(10)$ is symmetric in the sense that

$$
\mathcal{D}(w, r)=\mathcal{D}(r, w), \quad \forall w, r \in V_{h}
$$

REMARK 2.5. The operator $\mathcal{D}$ is symmetric for fluxes (10) with any $\theta \in[0,1]$ and any $\beta \in \mathbb{R}$. But as shown in [35] for suitably large $\beta>1$ we have

$$
\mathcal{D}(w, w) \geq 0
$$

which ensures that $\psi_{h}$ can be uniquely determined from solving (8) for given $u_{h}$. For more refined quantization of $\beta$, see [32].

REMARK 2.6. The symmetric property of operator $\mathcal{D}$ is consistent with the conservation induced by the dispersive effects. The entropy flux $\hat{f}$ plays the role of numerical diffusion, and serves to pick out the unique entropy shocks when discontinuous solutions present. In contrast, some non-symmetric $\mathcal{D}$ can well lead to false solutions.

2.3. Stability and conservative properties. In order to establish the conservation of $E_{2}$, we define $v_{h} \in V_{h}$ from a DG formulation of (4). Here we apply the DDG method to solve equation (4): from the numerical solution $u_{h} \in V_{h}$, we find $v_{h} \in V_{h}$, such that

$$
4\left(v_{h}, \eta\right)+\mathcal{D}\left(v_{h}, \eta\right)=\left(u_{h}, \eta\right), \quad \forall \eta \in V_{h},
$$

where the numerical fluxes are

$$
\hat{v}_{h x}=\beta \frac{\left[v_{h}\right]}{h}+v_{h x}^{\theta}, \quad \hat{v}_{h}=v_{h}^{1-\theta} .
$$

Existence of $v_{h}$ is guaranteed if $\beta$ is suitably large ( see the proof of the following theorem).

THEOREM 2.1. Let $u_{h}$ be the DDG solution and $v_{h}$ be defined in (21). Then we have

(i) Both invariants $E_{1}$ and $E_{2}$ are preserved for the DDG-C scheme in the sense that

$$
\frac{d}{d t} \int_{I} u_{h} d x=0, \quad \frac{d}{d t} \int_{I}\left(u_{h}^{2}-3 u_{h} v_{h}\right) d x=0
$$

(ii) For the DDG-D scheme, we have

$$
\frac{d}{d t} \int_{I} u_{h} d x=0, \quad \frac{d}{d t} \int_{I}\left(u_{h}^{2}-3 u_{h} v_{h}\right) d x \leq 0 .
$$

(iii) Both DDG-C and DDG-D schemes are $L^{2}$ stable:

$$
\left\|u_{h}(t)\right\| \leq \sqrt{7}\left\|u_{0}\right\|, \quad \forall t>0 .
$$


Proof. (i) To see the scheme preserves $E_{1}$ we simply take $w=r=1$ in (14)-(15) to obtain

$$
\begin{aligned}
\frac{d}{d t} \sum_{j=1}^{N} \int_{I_{j}} u_{h} d x & =-\mathcal{B}\left(u_{h}, 1\right)-\left(\psi_{h}, 1\right)=-\left(\psi_{h}, 1\right) \\
& =\mathcal{D}\left(\psi_{h}, 1\right)+3 \mathcal{B}\left(u_{h}, 1\right)=0 .
\end{aligned}
$$

To show the conservation of $E_{2}$, we first claim and later prove two identities

$$
\left(u_{h t}, u_{h}-3 v_{h}\right)=0
$$

and

$$
\left(u_{h t}, v_{h}\right)=\left(u_{h}, v_{h t}\right)
$$

These two relations ensure that

$$
\left(u_{h t}, u_{h}-3 v_{h}\right)=\frac{1}{2} \frac{d}{d t} \int_{I}\left(u_{h}^{2}-3 u_{h} v_{h}\right) d x=0 .
$$

It means that the quantity $E_{2}=\int_{I}\left(u_{h}^{2}-3 u_{h} v_{h}\right) d x$ is preserved by the DDG-C discretization:

$$
E_{2}=\int_{I}\left(u_{h}^{2}-3 u_{h} v_{h}\right) d x=E_{2}(0)
$$

(ii) For the DDG-D scheme, (19) yields

$$
\left(u_{h t}, u_{h}-3 v_{h}\right) \leq 0
$$

This together with (24) ensures that

$$
\frac{d}{d t} E_{2}=2\left(u_{h t}, u_{h}-3 v_{h}\right) \leq 0
$$

(iii) By choosing $\eta=v_{h}$ in equation (21) with the numerical fluxes (22), we have

$$
\begin{aligned}
\left(u_{h}, v_{h}\right) & =4\left\|v_{h}\right\|^{2}+\mathcal{D}\left(v_{h}, v_{h}\right) \\
& =4\left\|v_{h}\right\|^{2}+\left\|v_{h x}\right\|^{2}+\sum_{j}\left(\beta \frac{\left[v_{h}\right]^{2}}{h}+2 v_{h x}^{\theta}\left[v_{h}\right]\right)_{j-\frac{1}{2}} .
\end{aligned}
$$

As shown in [35], a suitably large $\beta>1$ can be made to ensure

$$
\left\|v_{h x}\right\|^{2}+\sum_{j}\left(\beta \frac{\left[v_{h}\right]^{2}}{h}+2 v_{h x}^{\theta}\left[v_{h}\right]\right)_{j-\frac{1}{2}} \geq 0 .
$$

Then we have $4\left\|v_{h}\right\|^{2} \leq\left(u_{h}, v_{h}\right) \leq\left\|u_{h}\right\|\left\|v_{h}\right\|$, hence

$$
\left\|v_{h}\right\| \leq \frac{1}{4}\left\|u_{h}\right\|
$$

From (26), we obtain

$$
\left\|u_{h}\right\|^{2}=E_{2}(t)+3\left(u_{h}, v_{h}\right) \leq E_{2}(0)+\frac{3}{4}\left\|u_{h}\right\|^{2} .
$$


Hence $\left\|u_{h}\right\|^{2} \leq 4 E_{2}(0)$. Using (30) we verify that $E_{2}(0) \leq \frac{7}{4}\left\|u_{0}\right\|^{2}$, so

$$
\left\|u_{h}\right\| \leq 2 \sqrt{E_{2}(0)} \leq \sqrt{7}\left\|u_{0}\right\|
$$

as desired.

We now conclude by verifying two claims (23) and (24), respectively. First we choose in equations (21), (14)-(15) test functions as

$$
\eta=\psi_{h}, \quad w=u_{h}-3 v_{h}, \quad r=-v_{h},
$$

so that

$$
\begin{aligned}
4\left(v_{h}, \psi_{h}\right)+\mathcal{D}\left(v_{h}, \psi_{h}\right)-\left(u_{h}, \psi_{h}\right) & =0, \\
\left(u_{h t}, u_{h}-3 v_{h}\right)+\mathcal{B}\left(u_{h}, u_{h}-3 v_{h}\right)+\left(\psi_{h}, u_{h}-3 v_{h}\right) & =0 \\
-\left(\psi_{h}, v_{h}\right)-\mathcal{D}\left(\psi_{h}, v_{h}\right)+3 \mathcal{B}\left(u_{h}, v_{h}\right) & =0 .
\end{aligned}
$$

Summing the above three equations together, we arrive at

$$
\left(u_{h t}, u_{h}-3 v_{h}\right)+\mathcal{B}\left(u_{h}, u_{h}\right)+\mathcal{D}\left(v_{h}, \psi_{h}\right)-\mathcal{D}\left(\psi_{h}, v_{h}\right)=0 .
$$

Taking into account the properties (18) and (20) of the two operators $\mathcal{B}$ and $\mathcal{D}$, we obtain (23). For the Lax-Friedrich flux we have (19), hence (27).

We next verify (24): we take the time derivative of (21) and choose test functions $\eta=v_{h}$ to have

$$
4\left(v_{h t}, v_{h}\right)+\mathcal{D}\left(v_{h t}, v_{h}\right)-\left(u_{h t}, v_{h}\right)=0 .
$$

We also choose the test function $\eta=v_{h t}$ in equation (21) to obtain

$$
4\left(v_{h}, v_{h t}\right)+\mathcal{D}\left(v_{h}, v_{h t}\right)-\left(u_{h}, v_{h t}\right)=0 .
$$

Taking the difference of (35) and (36), we obtain

$$
-\left(u_{h t}, v_{h}\right)+\mathcal{D}\left(v_{h t}, v_{h}\right)-\mathcal{D}\left(v_{h}, v_{h t}\right)+\left(u_{h}, v_{h t}\right)=0 .
$$

With the property $(20)$ of the operator $\mathcal{D}$, we easily obtain $(24)$.

2.4. Time discretization. We now turn to time discretization of (7)-(8). Let $\left\{t^{n}\right\}, n=0,1, \ldots, M$ be a uniform partition of the time interval $[0, T]$. Let $u_{h}^{0}=P u_{0}$ be the piecewise $L^{2}$ projection of $u_{0}(x)$. Given solution $u_{h}$ at a time level, we first solve (15) to get $\psi_{h}$

$$
\psi_{h}=M\left(u_{h}\right) .
$$

Using solution $\psi_{h}$ to compute solution $u_{h}$ in (14), then we obtain

$$
\left(u_{h}\right)_{t}=L\left(u_{h}, \psi_{h}\right)=L\left(u_{h}, M\left(u_{h}\right)\right)
$$

We use the third order explicit Runge-Kutta method for time discretization. In details, let $u_{h}^{n}$ be the solution at time level $n$,

$$
\begin{aligned}
u_{h}^{(0)} & =u_{h}^{n} \\
u_{h}^{(i)} & =\sum_{j=0}^{i-1}\left(a_{i j} u_{h}^{(j)}+b_{i j} \Delta t L\left(u_{h}^{(j)}, M\left(u_{h}^{(j)}\right)\right)\right), \quad i=1,2,3 ; \\
u_{h}^{n+1} & =u_{h}^{(3)}
\end{aligned}
$$


where

$$
\begin{aligned}
& a_{10}=b_{10}=1, \quad a_{20}=\frac{3}{4}, \quad b_{20}=0, \quad a_{21}=b_{21}=\frac{1}{4}, \\
& a_{30}=\frac{1}{3}, \quad b_{30}=a_{31}=b_{31}=0, \quad a_{32}=b_{32}=\frac{2}{3} .
\end{aligned}
$$

3. Numerical examples. One of the features of the DP equation, as for the $\mathrm{CH}$ equation, is that it admits a class of weak solutions known as peakons. A peakon (peaked soliton) is a wave of the form $u=m_{0} G\left(x-x_{0}\right)$ where

$$
G(x)=e^{-|x|}
$$

The $n$-peakon solution of the DP equation is a superposition of interacting peakons,

$$
u(x, t)=\sum_{j=1}^{n} m_{j}(t) G\left(x-x_{j}(t)\right)
$$

whose positions $x_{j}(t)$ and momenta $m_{j}(t)$ are governed by the

$$
\dot{x}_{j}=u\left(x_{j}\right), \quad \dot{m}_{j}=-2 m_{j} u_{x}\left(x_{j}\right) .
$$

The DP peakon ODE system can be solved explicitly in terms of elementary functions, see $[39,40]$. For example, it admits a shock peakon solution of the form

$$
u(x, t)=-\frac{1}{1+t} \operatorname{sign}(x) e^{-|x|}
$$

We shall present different combinations of peakons and test their evolution and interactions.

We also verify the conservative property of the DDG method, and compare it with a non-conservative DDG method. As mentioned in Remark 2.2 earlier, DDG-C is used to denote our DDG method with conservative numerical fluxes

(41)

$$
\hat{f}\left(u_{h}\right)=\frac{\int_{u_{h}^{-}}^{u_{h}^{+}} f(u) d u}{\left[u_{h}\right]}, \quad \hat{\psi}_{h x}=\beta \frac{\left[\psi_{h}\right]}{h}+\theta \psi_{h x}^{+}+(1-\theta) \psi_{h x}^{-}, \quad \hat{\psi}_{h}=(1-\theta) \psi_{h}^{+}+\theta \psi_{h}^{-} .
$$

The DDG-D method denotes the DDG method with the dissipative numerical fluxes

$$
\begin{aligned}
& \hat{f}\left(u_{h}^{-}, u_{h}^{+}\right)=\frac{1}{2}\left(f\left(u_{h}^{-}\right)+f\left(u_{h}^{+}\right)-\sigma\left(u_{h}^{+}-u_{h}^{-}\right)\right), \\
& \hat{\psi}_{h x}=\beta \frac{\left[\psi_{h}\right]}{h}+\theta \psi_{h x}^{+}+(1-\theta) \psi_{h x}^{-}, \quad \hat{\psi}_{h}=(1-\theta) \psi_{h}^{+}+\theta \psi_{h}^{-},
\end{aligned}
$$

where $\sigma=\max _{u \in\left(u_{h}^{-}, u_{h}^{+}\right)}\left|f^{\prime}(u)\right|$.

ExAmPle 3.1. Accuracy test

The peaked traveling wave solution is

$$
u(x, t)=c e^{-|x-c t|},
$$

where $c=0.25$ is the wave speed. The accuracy is measured in the smooth parts of the solution, 1/20 of the computational domain away from the peak. We test the 
$P^{k}$ polynomial approximation on uniform meshes. The $L^{2}$ errors and the numerical orders of accuracy for $k=0,1,2,3,4$ with different $\theta=0,1 / 2,1$ at time $t=1$ are reported in Table 1. We can clearly see that the DDG-C methods achieve the optimal convergence rates for $P^{k}$ elements. Different choices of $\theta$ are shown to yield the same order of accuracy. Thus in the following examples, we simply take the numerical fluxes (41) or (42) with only $\theta=0$.

TABLE 1

Accuracy test of DDG-C for the DP equation with the exact solution (43). Periodic bounday condition, $c=0.25$, uniform meshes with $N$ cells at time $t=1$.

\begin{tabular}{|c|c|c|c|c|c|c|c|c|c|}
\hline & \multicolumn{3}{|c|}{$\theta=0$} & \multicolumn{3}{|c|}{$\theta=1 / 2$} & \multicolumn{3}{|c|}{$\theta=1$} \\
\hline & $\mathrm{N}$ & $L^{2}$ error & order & $\mathrm{N}$ & $L^{2}$ error & order & $\mathrm{N}$ & $L^{2}$ error & order \\
\hline \multirow{4}{*}{$P^{0}$} & 20 & $3.5588 \mathrm{e}-02$ & - & 20 & $3.5588 \mathrm{e}-02$ & - & 20 & $3.5588 \mathrm{e}-02$ & - \\
\hline & 40 & $9.55647 \mathrm{e}-03$ & 1.91 & 40 & $9.55647 \mathrm{e}-03$ & 1.91 & 40 & $9.55647 \mathrm{e}-03$ & 1.91 \\
\hline & 80 & $4.9544 \mathrm{e}-03$ & 1.21 & 80 & $4.9544 \mathrm{e}-03$ & 1.21 & 80 & $4.9544 \mathrm{e}-03$ & 1.21 \\
\hline & 160 & $2.08456 \mathrm{e}-03$ & 0.97 & 160 & $2.08456 \mathrm{e}-03$ & 0.97 & 160 & $2.08456 \mathrm{e}-03$ & 0.97 \\
\hline \multirow{4}{*}{$P^{1}$} & 20 & $1.35755 \mathrm{e}-02$ & - & 20 & $1.36711 \mathrm{e}-02$ & - & 20 & $1.37979 \mathrm{e}-02$ & - \\
\hline & 40 & $2.02881 \mathrm{e}-03$ & 2.74 & 40 & $2.043 \mathrm{e}-03$ & 2.74 & 40 & $2.01756 \mathrm{e}-03$ & 2.77 \\
\hline & 80 & $3.64350 \mathrm{e}-04$ & 2.48 & 80 & $3.65808 \mathrm{e}-04$ & 2.48 & 80 & $3.61886 \mathrm{e}-04$ & 2.48 \\
\hline & 160 & $8.31758 \mathrm{e}-05$ & 2.13 & 160 & $8.30873 \mathrm{e}-05$ & 2.14 & 160 & $8.26638 \mathrm{e}-05$ & 2.13 \\
\hline \multirow{4}{*}{$P^{2}$} & 20 & $2.23816 \mathrm{e}-03$ & - & 20 & $1.03916 \mathrm{e}-03$ & - & 20 & $1.07614 \mathrm{e}-03$ & - \\
\hline & 40 & $1.75352 \mathrm{e}-04$ & 3.67 & 40 & $2.02092 \mathrm{e}-04$ & 2.36 & 40 & $2.01937 \mathrm{e}-04$ & 2.42 \\
\hline & 80 & $1.85993 \mathrm{e}-05$ & 3.24 & 80 & $1.71086 \mathrm{e}-05$ & 3.56 & 80 & $1.7065 \mathrm{e}-05$ & 3.56 \\
\hline & 160 & $2.22274 \mathrm{e}-06$ & 3.06 & 160 & $2.00908 \mathrm{e}-06$ & 3.09 & 160 & $2.00661 \mathrm{e}-06$ & 3.09 \\
\hline \multirow{4}{*}{$P^{3}$} & 20 & $2.06030 \mathrm{e}-03$ & - & 20 & $2.07289 \mathrm{e}-03$ & - & 20 & $1.98868 \mathrm{e}-03$ & - \\
\hline & 40 & $1.35896 \mathrm{e}-04$ & 3.92 & 40 & $1.34196 \mathrm{e}-04$ & 3.95 & 40 & $1.32673 \mathrm{e}-04$ & 3.91 \\
\hline & 80 & $1.11821 \mathrm{e}-06$ & 6.93 & 80 & $1.10354 \mathrm{e}-06$ & 6.93 & 80 & $1.08481 \mathrm{e}-06$ & 6.93 \\
\hline & 160 & $6.39321 \mathrm{e}-08$ & 4.13 & 160 & $6.708997 \mathrm{e}-08$ & 4.04 & 160 & $6.7157 \mathrm{e}-08$ & 4.01 \\
\hline \multirow{4}{*}{$P^{4}$} & 20 & $1.93881 \mathrm{e}-03$ & - & 20 & $1.94255 \mathrm{e}-03$ & - & 20 & $1.90279 \mathrm{e}-03$ & - \\
\hline & 40 & $1.63162 \mathrm{e}-04$ & 3.57 & 40 & $1.62779 \mathrm{e}-04$ & 3.58 & 40 & $1.62189 \mathrm{e}-04$ & 3.55 \\
\hline & 80 & $2.62141 \mathrm{e}-06$ & 5.96 & 80 & $2.62063 \mathrm{e}-06$ & 5.96 & 80 & $2.61642 \mathrm{e}-06$ & 5.95 \\
\hline & 160 & $1.53566 \mathrm{e}-08$ & 7.42 & 160 & $1.65437 \mathrm{e}-08$ & 7.31 & 160 & $1.65379 \mathrm{e}-08$ & 7.31 \\
\hline
\end{tabular}

EXAmple 3.2. Peakon solution

In this example, we present the wave propagation of the peakon solution

$$
u(x, t)=c e^{-|x-c t|},
$$

where $c=1$ is the wave speed. The computational domain is $[-40,40]$. We use the $P^{4}$ element with $N=228$ cells to resolve the peak. Figure 1 shows the plots of the $E_{1}, E_{2}$ and $E_{3}$ from $t=0$ to $t=1000$ of the two methods, DDG-C and DDG-D respectively. According to Theorem 2.1, the DDG-C scheme conserves $E_{1}$ and $E_{2}$. We confirmed that the DDG-C scheme preserves these two invariants, while the DDG-D scheme actually does not conserve $E_{2}$ and $E_{3}$, and the dissipation is clearly observed. On the other hand, Figure 1 shows that the $D D G$-C scheme almost preserves the value of $E_{3}$ even though this has not been verified in our theoretical analysis. Figure 2 plots the peakon solutions of the DDG-C method and DDG-D method at $t=0,100,250,520,740$ and 1000. The exact solution is provided as a reference in the plot. The DDG-D method has a large phase error, while the DDG-C method method gives quite a good approximation to the exact solution.

EXAMPLE 3.3. Anti-peakon solution

In this example, we present the wave propagation of the anti-peakon solution

$$
u(x, t)=c e^{-|x-c t|},
$$




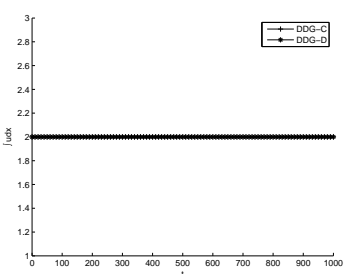

$E_{1}(u)=\int_{I} u d x$

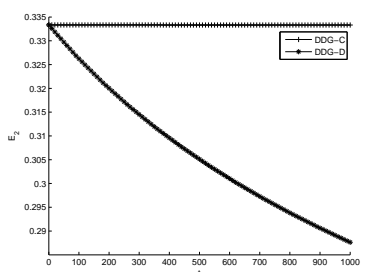

$E_{2}(u)=\int_{I}\left(u^{2}-3 u v\right) d x$

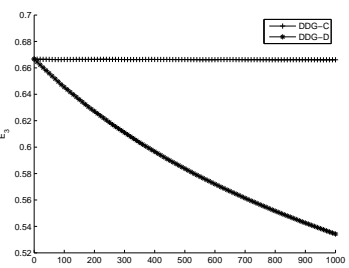

$E_{3}(u)=\int_{I} u^{3} d x$

FIG. 1. Example 3.2: Time history of the $E_{1}, E_{2}$ and $E_{3}$ plots using DDG-C and DDG-D methods. $P^{4}$ elements and a uniform mesh with $N=228$ cells.

where $c=-1$ is the wave speed. The computational domain is $[-40,40]$. We use the $P^{4}$ element with $N=228$ cells to resolve the peak. The numerical results were qualitatively entirely consistent with those in Example 3.2. We plot in Figure 3 the values of $E_{1}, E_{2}, E_{3}$ from $t=0$ to $t=1000$ of the two methods, DDG-C and DDG$D$ respectively. We confirmed that the $D D G-C$ method preserves the values of $E_{1}$ and $E_{2}$, and almost preserves the value of $E_{3}$, but the $D D G-D$ method conserves only $E_{1}$, and the dissipation is clearly observed. In Figure 4, the peakon profile at $t=0,100,250,520,740$ and 1000 are shown. Again, one observes a large phase error in the DDG-D solution, while the DDG-C method match the exact solution very well.

EXAMPLE 3.4. Two-peakon interaction

In this example we consider the two-peakon interactions [22, 38, 47] of the DP equation with the initial condition

$$
u_{0}(x)=\phi_{1}(x)+\phi_{2}(x), \quad \phi_{i}(x)=c_{i} e^{-\left|x-x_{i}\right|}, i=1,2,
$$

where $c_{1}=2, c_{2}=1, x_{1}=-13.792, x_{2}=-4$. The computational domain is $[-40,40]$. We use the $P^{3}$ element with $N=512$ cells in our computation by the DDG methods. Figure 5 shows the time evolution of the values $E_{1}, E_{2}, E_{3}$ up to time $t=200$ of the two methods, DDG-C and DDG-D respectively. We see clearly that the DDG-C scheme preserves $E_{1}, E_{2}$, while the $D D G$-D scheme does not conserve $E_{2}, E_{3}$, and the dissipation is clearly observed. Figure 6 plots the snapshots at different times, together with the exact solution. We see that the moving peakon interaction is resolved very well.

EXAMPLE 3.5. Two-anti-peakon interaction

In this example we consider the two-anti-peakon interaction [22, 38, 47] of the $D P$ equation with the initial condition

$$
u_{0}(x)=\psi_{1}(x)+\psi_{2}(x), \quad \psi_{i}(x)=-c_{i} e^{-\left|x+x_{i}\right|}, i=1,2,
$$

where $c_{1}=2, c_{2}=1, x_{1}=-13.792, x_{2}=-4$. The computational domain is $[-40,40]$. We use the $p^{3}$ element with $N=512$ cells in our computation by the DDG method. The values of $E_{2}, E_{3}$ up to time $t=200$ are shown in Figure 7 . We confirmed that the $D D G$-C scheme preserves $E_{1}$ and $E_{2}$, but the $D D G$-D scheme actually does not conserve $E_{2}, E_{3}$. Figure 8 shows the numerical solutions by DDG-C method and DDG$D$ method at $t=0,10,100$ and 200. Similar to the Example 3.4, better performance of the DDG-C method is observed. 


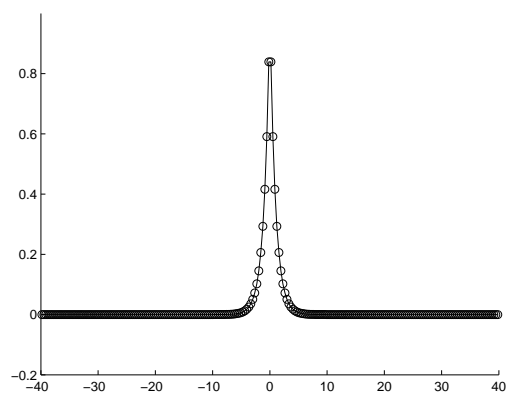

$\mathrm{t}=0$

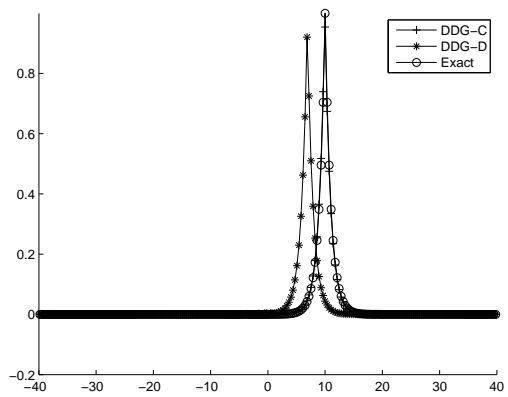

$\mathrm{t}=250$

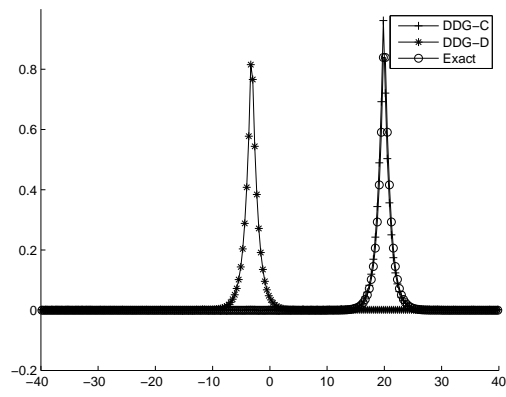

$\mathrm{t}=740$

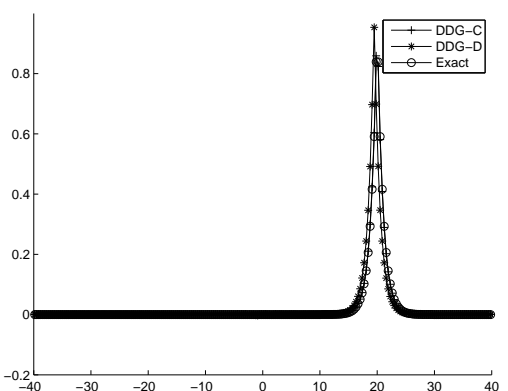

$\mathrm{t}=100$

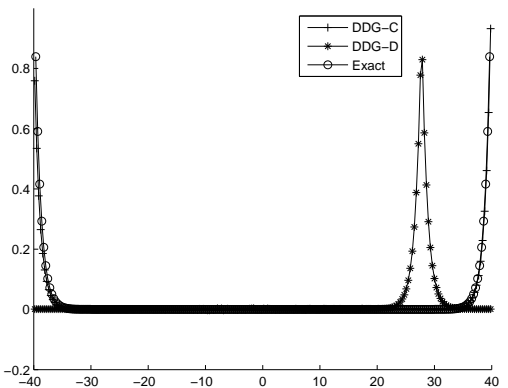

$\mathrm{t}=520$

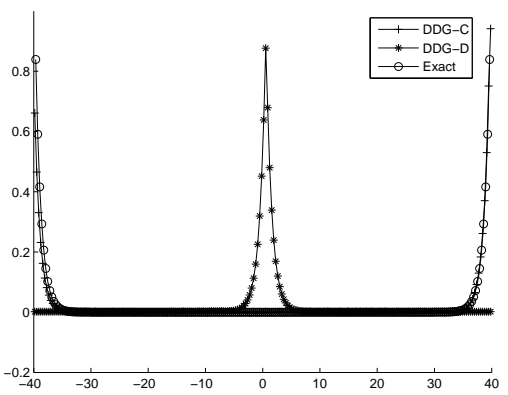

$\mathrm{t}=1000$

FIG. 2. Example 3.2: The peakon profile of the DP equation with the exact solution (44). Periodic boundary condition in $[-40,40] . P^{4}$ elements and a uniform mesh with $N=228$ cells.

From the above four examples, we observe that, compared to the non-conservative scheme DDG-D, the conservative scheme DDG-C preserves the values of $E_{1}, E_{2}$, and almost preserves the value $E_{3}$, which is entirely consistent with the theoretical results. We also note that, in the long-time simulation, the numerical solution by DDG-C method has smaller phase error. This implies the DDG-C method is perferred over the DDG-D method. Therefore we conclude that the conservative scheme DDG-C is more efficient.

To compare with existing numerical methods of the DP equation, we further test the proposed DDG method in capturing the shock solutions of the DP equation. In 


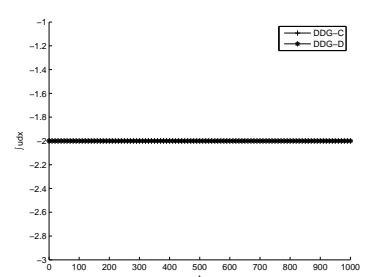

$E_{1}(u)=\int_{I} u d x$

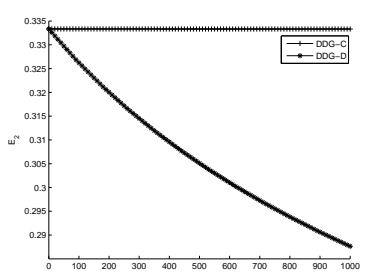

$E_{2}(u)=\int_{I}\left(u^{2}-3 u v\right) d x$

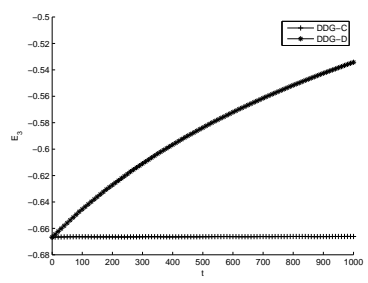

$E_{3}(u)=\int_{I} u^{3} d x$

FIG. 3. Example 3.3: Time history of the $E_{1}, E_{2}$ and $E_{3}$ plots using $D D G-C$ and $D D G-D$ methods. $P^{4}$ elements and a uniform mesh with $N=228$ cells.

what follows, we choose the adaptive numerical fluxes in order to capture the strong shocks,

$$
\begin{gathered}
\hat{f}\left(u^{-}, u^{+}\right)=\left\{\begin{array}{cl}
\frac{\int_{u^{-}}^{u^{+}} f(u) d u}{[u]}, & \text { if }\left|u^{+}-u^{-}\right| \leq 10^{-2}, \\
\frac{1}{2}\left(f\left(u^{-}\right)+f\left(u^{+}\right)-\epsilon\left(u^{+}-u^{-}\right)\right), & \text {otherwise, }
\end{array}\right. \\
\sigma=\max _{u \in\left(u_{h}^{-}, u_{h}^{+}\right)}\left|f^{\prime}(u)\right|, \quad \hat{\psi}_{x}=\beta \frac{[\psi]}{h}+\psi_{x}^{-}, \quad \hat{\psi}=\psi^{+} .
\end{gathered}
$$

Here we set $10^{-2}$ as the shock detector. We use the TVBM limiter [6] to remove oscillation near any discontinuity of $u$.

EXAMPLE 3.6. Shock peakon solution

In this example, we present the wave evolution of the shock peakon solution [47]

$$
u(x, t)=-\frac{1}{1+t} \operatorname{sign}(x) e^{-|x|}
$$

The computational domain is $[-30,30]$. In our computation by the DDG method, we use the $P^{4}$ element with $N=228$ cells to resolve the shock peakon. In Figure 9, the shock peakon profile at $t=0,1,2,6$ are shown. We see clearly that the numerical solution agrees quite well with the exact one. The shock interface is very sharp, and there is no numerical oscillation observed for the proposed DDG method. The results agree with those obtained in [47].

EXAMPLE 3.7. Shock formation

In this example, we consider the shock formation [9, 22, 47] of DP equation with the initial condition

$$
u_{0}(x)=e^{0.5 x^{2}} \sin (\pi x) .
$$

The computational domain is $[-2,2]$. We perform computation on 100 cells with $P^{4}$ elements. The numerical solutions at four different times $t=0,0.18,0.5,1.1$ are plotted in Figure 10. The shock interface is very sharp. There are only two transition points in the position of shocks and no numerical oscillation is observed. The results agree with those in [9, 22, 47].

We also numerically investigate the dissipation of $E_{2}$ in the presence of shocks with the three schemes, denoted by DDG-M,DDG-A and DDG-L, respectively. These 

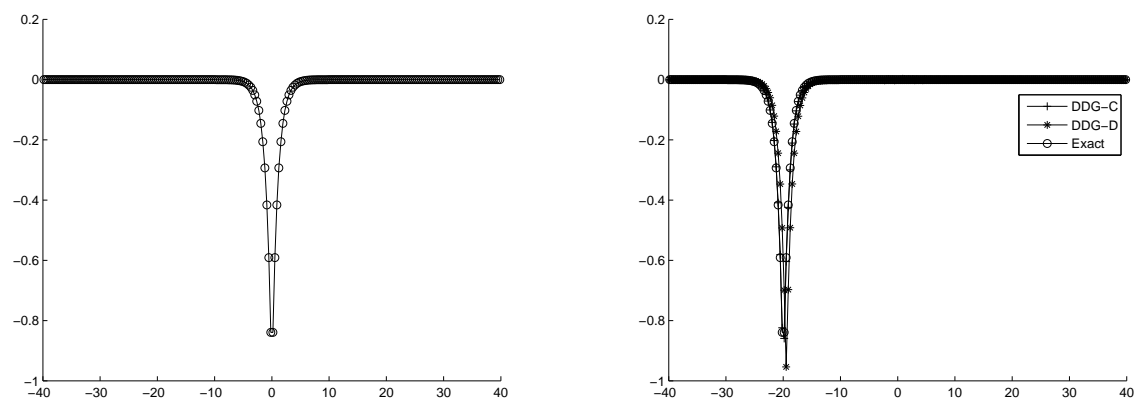

$\mathrm{t}=0$

$\mathrm{t}=100$
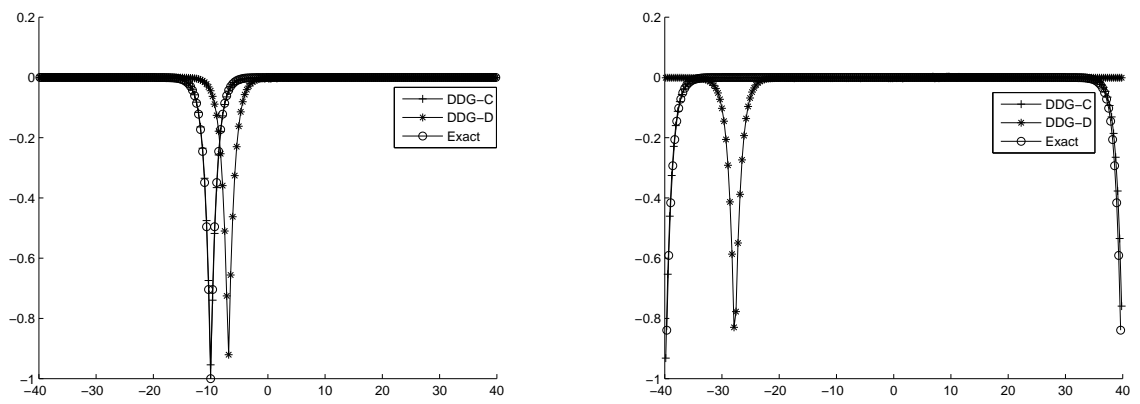

$\mathrm{t}=250$

$\mathrm{t}=520$

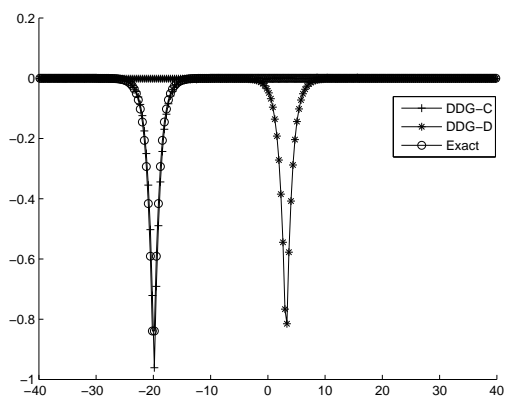

$\mathrm{t}=740$

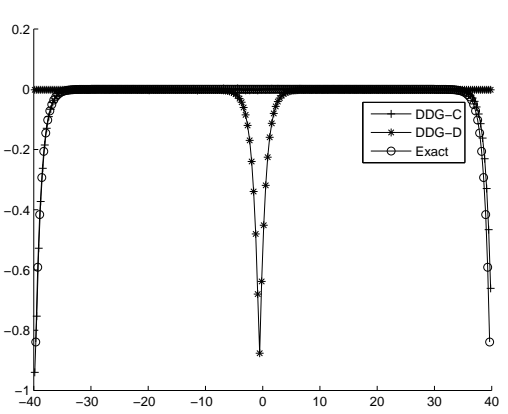

$\mathrm{t}=1000$

FIG. 4. Example 3.3: The peakon profile of the DP equation with the exact solution (45). Periodic boundary condition in $[-40,40] . P^{4}$ elements and a uniform mesh with $N=228$ cells.

schemes reflect only different choices of the numerical flux $\hat{f}(u)$ and the way of using the TVBM limiter. DDG-M corresponds to the modified numerical flux (48), with no TVBM limiter before the time $t=0.15 ; D D G$-A still corresponds to the modified numerical flux (48), but with the TVB limiter all the time; and DDG-L corresponds to the local Lax-Friedrich flux (42), also with the TVBM limiter all the time. Figure 11 shows the time evolution $E_{2}$ up to time $t=1.1$ of the three schemes. The dissipation of $E_{2}$ with different numerical flux $\hat{f}$ looks almost the same. The right figure is the local view from $t=0$ to $t=0.16$. We can see that the $D D G$ - $M$ preserves $E_{2}$ before the shock is formed. 


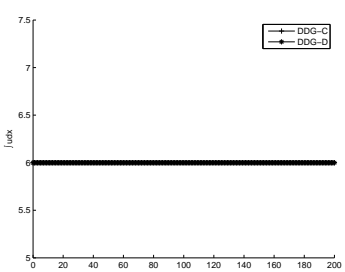

$E_{1}(u)=\int_{I} u d x$

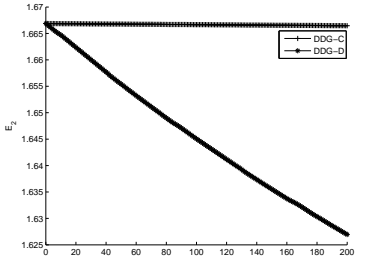

$E_{2}(u)=\int_{I}\left(u^{2}-3 u v\right) d x$

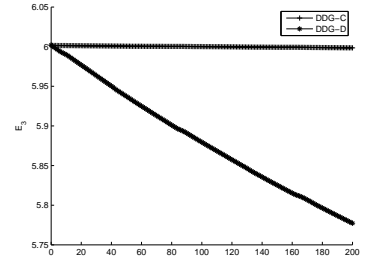

$E_{3}(u)=\int_{I} u^{3} d x$

FIG. 5. Example 3.4: Time history of the $E_{1}, E_{2}$ and $E_{3}$ plots using $D D G-C$ and $D D G-D$ methods. $P^{3}$ elements and a uniform mesh with $N=512$ cells.

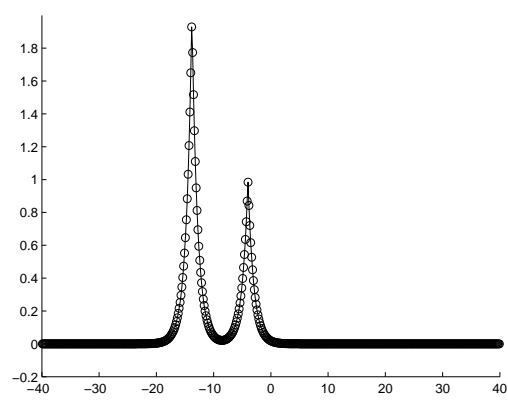

$\mathrm{t}=0$

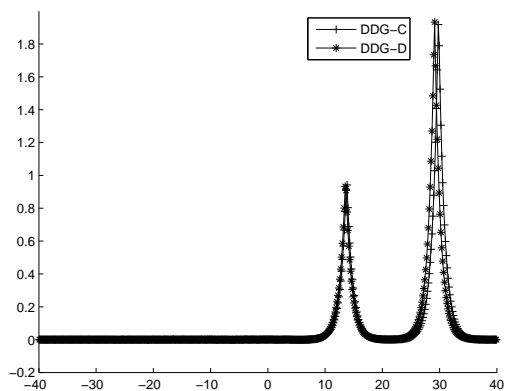

$\mathrm{t}=100$

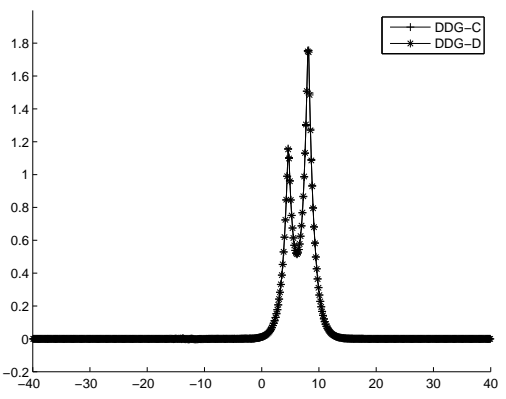

$\mathrm{t}=10$

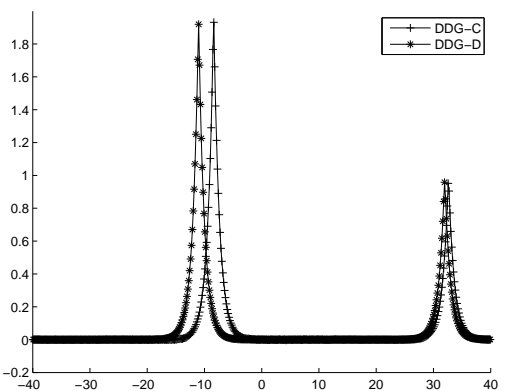

$t=200$

FIG. 6. Example 3.4: The peakon profile of the DP equation with the initial condition (46). Periodic boundary condition in $[-40,40] . P^{3}$ elements and a uniform mesh with $N=512$ cells.

EXAMPLE 3.8. Symmetric peak and anti-peak interaction

In this example, we consider the symmetric peak and anti-peak interaction [22, 38, 47] of DP equation with initial condition

$$
u_{0}(x)=e^{-|x+5|}-e^{-|x-5|} .
$$

The computational domain is $[-20,20]$. We use $P^{4}$ element with $N=256$ cells in our computation by the DDG method. The numerical solution at four different times are plotted in Figure 12. The interaction process can be visualized from a series of snapshots: $t=0$ (two peakon), $t=4$ (before collision), 5 (collision), 7 (after collision, one shock peakon). We see that a stationary shock peakon is formed at 


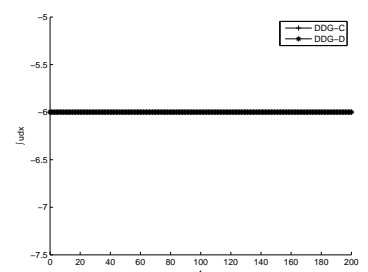

$E_{1}(u)=\int_{I} u d x$

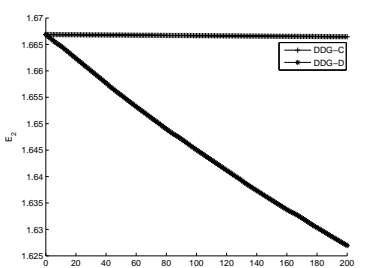

$E_{2}(u)=\int_{I}\left(u^{2}-3 u v\right) d x$

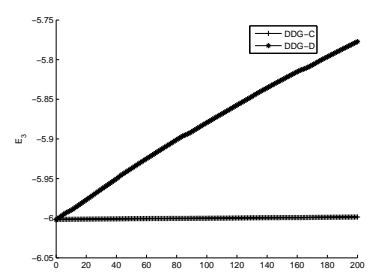

$E_{3}(u)=\int_{I} u^{3} d x$

FIG. 7. Example 3.5: Time history of the $E_{1}, E_{2}$ and $E_{3}$ plots using $D D G-C$ and $D D G-D$ methods. $P^{3}$ elements and a uniform mesh with $N=512$ cells.

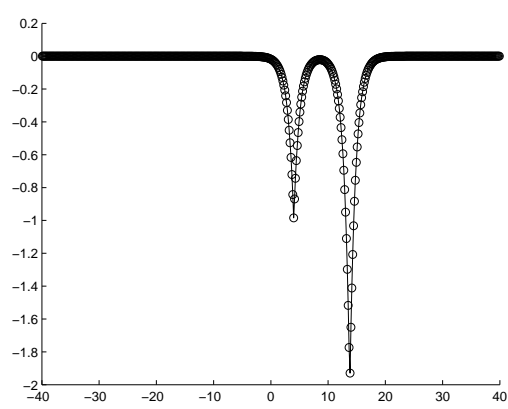

$\mathrm{t}=0$

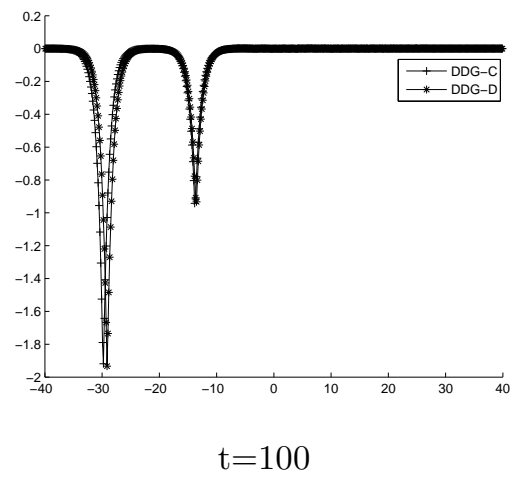

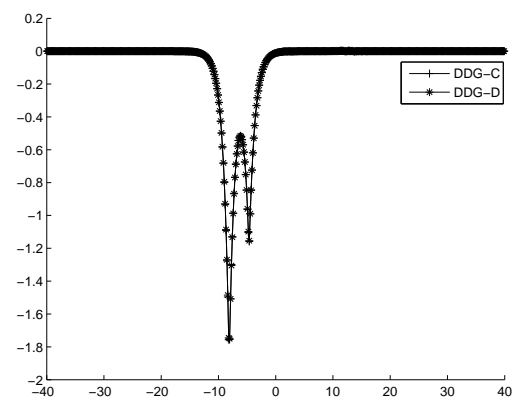

$\mathrm{t}=10$

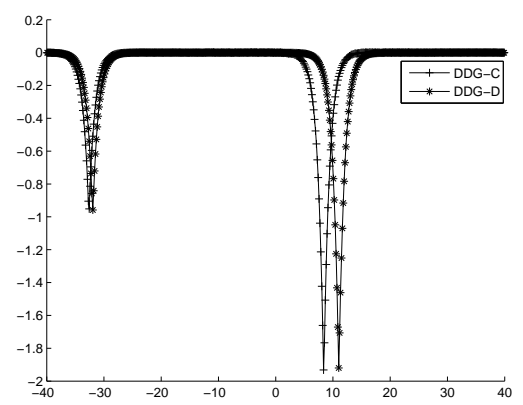

$\mathrm{t}=200$

FIG. 8. Example 3.5: The peakon profile of the DP equation with the initial condition (47). Periodic boundary condition in $[-40,40] . P^{3}$ elements and a uniform mesh with $N=512$ cells.

$t \approx 5$ and evolution follows one shock peakon solution. The interaction is resolved very well. The DDG method can automatically capture the shock and shock interface is very sharp. There is no numerical oscillation during the peakon and anti-peakon interaction.

EXAMPLE 3.9. Nonsymmetric peak and anti-peak interaction

In this example, we consider the nonsymmetric peak and anti-peak interaction [22, 38, 47] of DP equation with initial condition

$$
u_{0}(x)=2 e^{-|x+5|}-e^{-|x-5|} .
$$




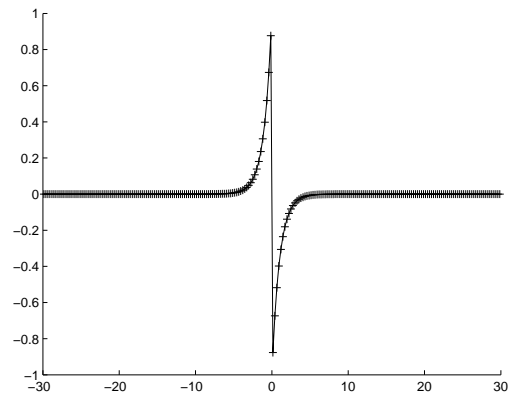

$\mathrm{t}=0$

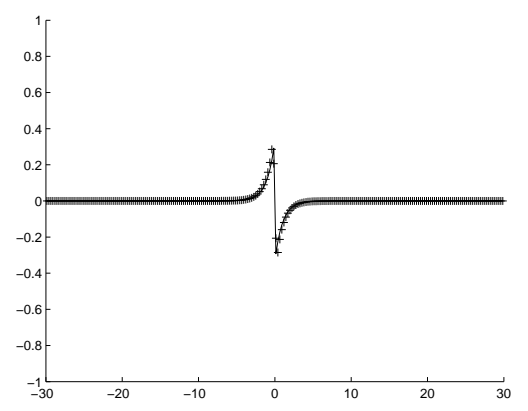

$\mathrm{t}=2$

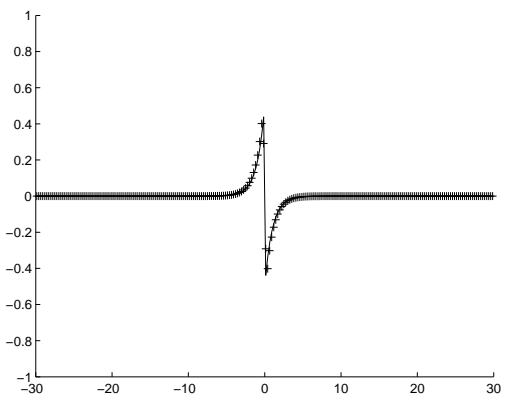

$\mathrm{t}=1$

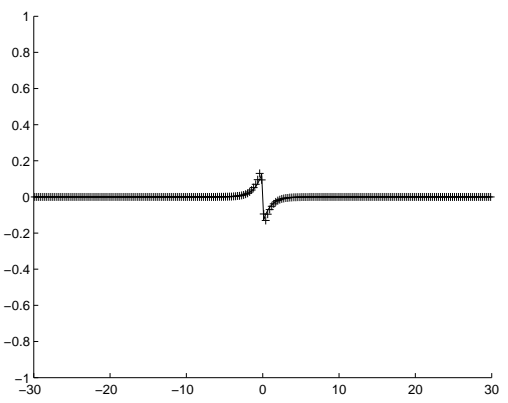

$\mathrm{t}=6$

FIG. 9. Example 3.6: Shock peakon solution of DP equation with the exact solution (49). Periodic boundary condition in $[-30,30] . P^{4}$ elements and a uniform mesh with $N=228$ cells.

The computational domain is $[-25,25]$. We use $P^{4}$ element with $N=256$ cells in our computation by the DDG method. Initially, a peakon of amplitude 2.0 and an antipeakon of amplitude 1.0 are located at $x_{1}=-5.0$ and $x_{2}=5.0$, respectively. Based on the results by Lundmark [38], two peakon solutions hold until $t<t_{c}=3.3626$. At $t=t_{c}$, a moving shock peakon is formed. Figure 13 shows the snapshots of the numerical solution before and after the collision. It shows that all of them agree quite well with the numerical result obtained by Xu and Shu [47]. We can see that a stationary shock peakon is formed at $t \approx 3.362$ and evolution follows one shock peakon solution. The interaction is resolved very well. The DDG method can automatically capture the shock and there is no numerical oscillation during the peakon and antipeakon interaction.

EXAMPLE 3.10. Triple interaction

In this example, we consider the peakon, shock peakon and anti-peakon interaction of DP equation with initial condition

$$
u_{0}(x)=e^{-|x+5|}+\operatorname{sign}(x) e^{-|x|}-e^{-|x-5|} .
$$

The computational domain is $[-25,25]$. We use $P^{4}$ element with $N=256$ cells in our computation by the DDG method. This triple collision was studied theoretically in [38] and numerically in [9, 22, 47]. Figure 14 displays the snapshots at different times. We observed the similar phenomenon as those in [47]. We see clearly that the interaction is resolved very well and shock interface is very sharp. 


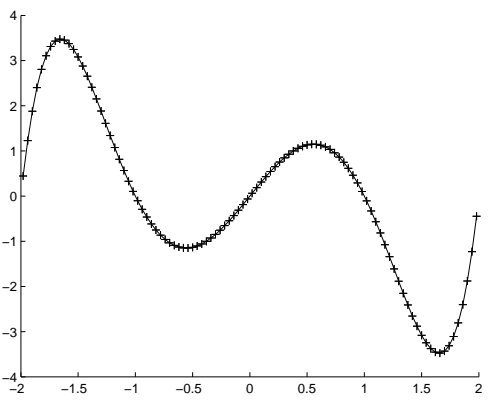

$\mathrm{t}=0$

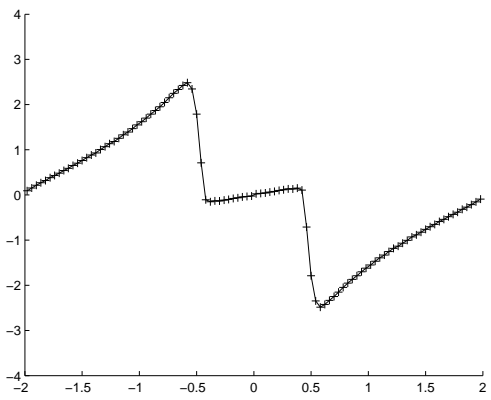

$\mathrm{t}=0.5$

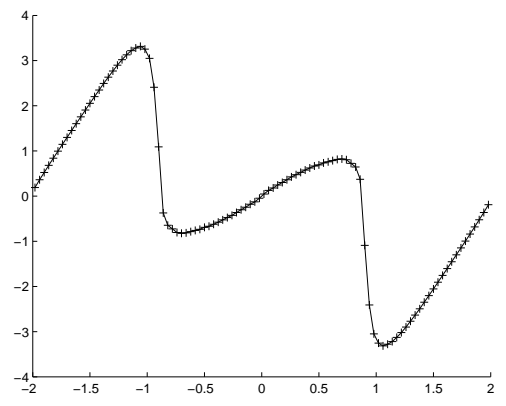

$\mathrm{t}=0.18$

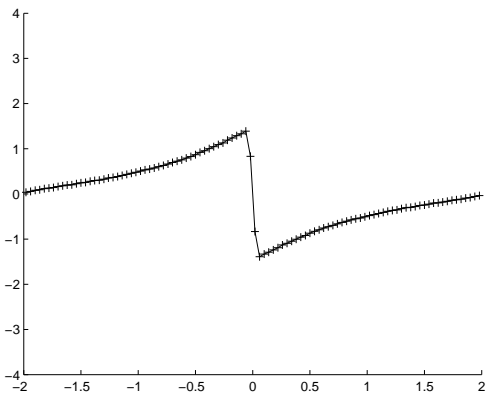

$\mathrm{t}=1.1$

FIG. 10. Example 3.7: Shock peakon solution of DP equation with the initial condition (50). Periodic boundary condition in $[-2,2] . P^{4}$ elements and a uniform mesh with $N=100$ cells.
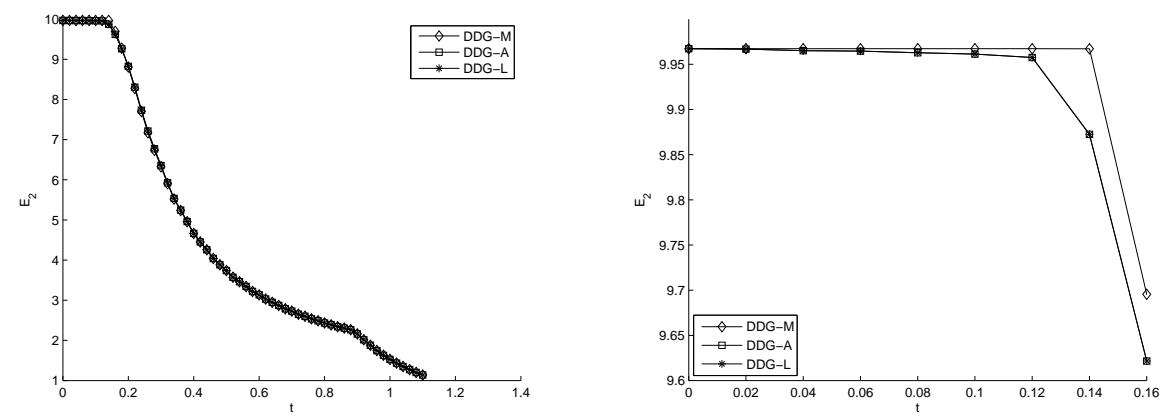

FIG. 11. Example 3.7: Time history of the $E_{2}$ plots. $P^{3}$ elements and a uniform mesh with $N=512$ cells.

Acknowledgments. Liu's research was partially supported by DMS09-07963 and DMS13-12636. Huang's research was supported in part by the NSFC Key Project (11031006), Hunan Provincial NSF Project (10JJ7001), and Hunan Education Department Key Project (10A117). Yi's research was supported by NSFC Project (11201397), Hunan Education Department Project (12B127), and Hunan Provincial NSF Project (12JJ4004) 

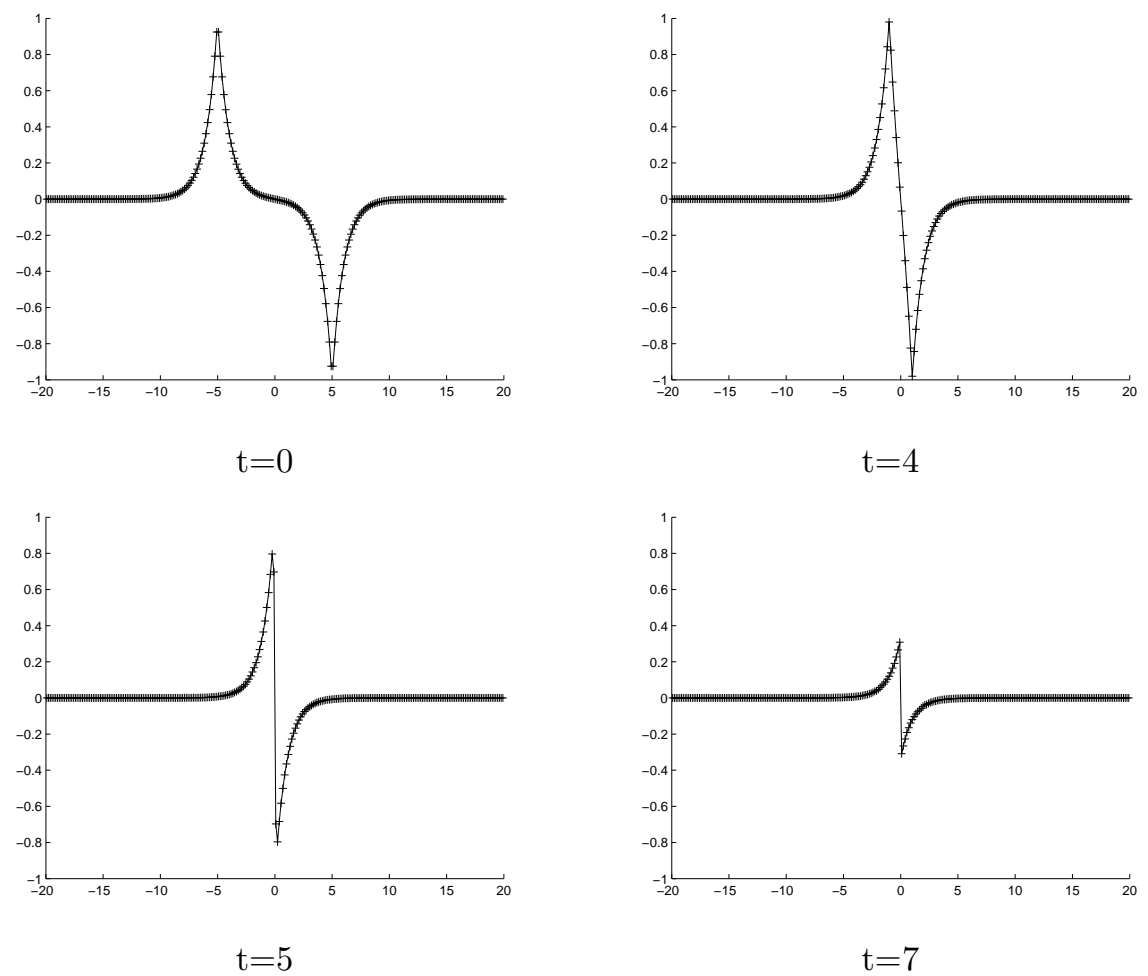

FIG. 12. Example 3.8: Symmetric peak and anti-peak interaction of DP equation with the initial condition (51). Periodic boundary condition in $[-20,20] . P^{4}$ elements and a uniform mesh with $N=256$ cells.

\section{REFERENCES}

[1] J. Bona, H. Chen, O. Karakashian, And Y. Xing, Conservative, discontinuous-Galerkin methods for the generalized Korteweg-de Vries equation, Math. Comp., 82 (2013), pp. 1401-1432.

[2] Y. Cheng And C. W. Shu, A discontinuous Galerkin finite element method for time dependent partial differential equations with higher order derivatives, Math. Comp., 77 (2008), pp. 699-730.

[3] R. Camassa And D. D. Holm, An integrable shallow water equation with peaked solitons, Physical Review Letters, 71 (1993), pp. 1661-1664.

[4] R. Camassa, D. D. Holm, and J. M. Hyman, A new integrable shallow water equation, Advances in Applied Mechanics, 31 (1994), pp. 1-33.

[5] E. Celledoni, V. Grimm, R. I. Mclachlan, D. I. Mclaren, D. O'Neale, B. Owren, and G. R. W. QUISPEL, Preserving energy resp. dissipation in numerical pdes using the average vector field method, J. Computational Physics, 231 (2012), pp. 6770-6789.

[6] B. Cockburn and C. W. Shu, TVB Runge-Kutta local projection discontinuous Galerkin finite element method for conservation laws II: general framework, Math. Comp., 52 (1989), pp. 411-435.

[7] G. Coclite And K. Karlsen, On the well-posedness of the Degasperis-Procesi equation, J. Funct. Anal., 233 (2006), pp. 60-91.

[8] G. Coclite and K. Karlsen, On the uniqueness of discontinuous solutions to the DegasperisProcesi equation, J. Differential Equations, 234 (2007), pp. 142-160.

[9] G. Coclite, K. Karlsen, And N. Risebro, Numerical schemes for computing discontinuous solutions of the Degasperis-Procesi equation, IMA J. Numer. Anal., 28 (2008), pp. 80-105.

[10] G. M. Coclite, F. Gargano, and V. Sciacca, Analytic solutions and singularity formation for the peakon b-family equations, Acta Applicandae Mathematicae, 122 (2012), pp. 419- 


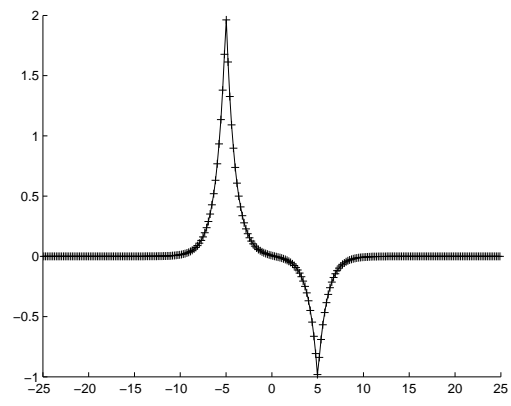

$\mathrm{t}=0$

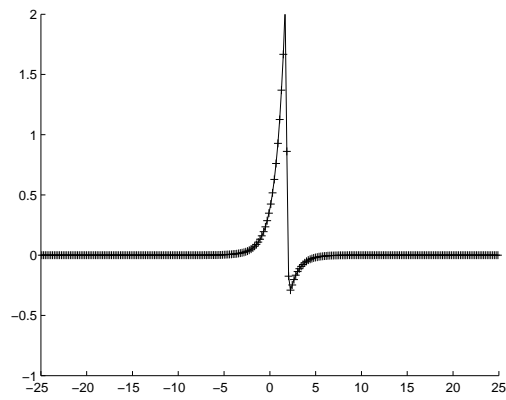

$\mathrm{t}=3.362$

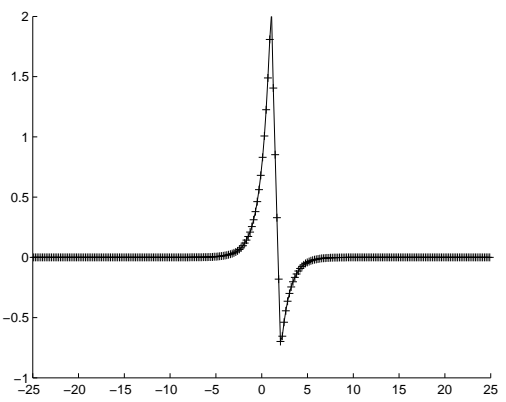

$\mathrm{t}=3$

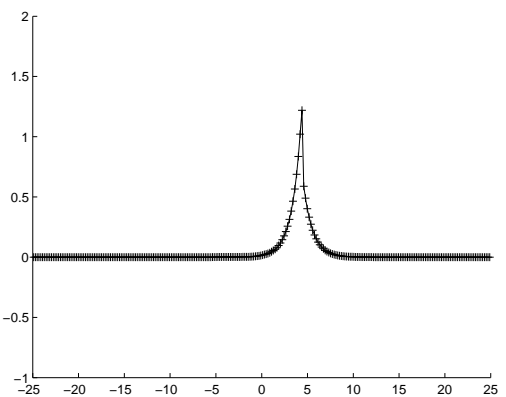

$\mathrm{t}=6$

FIG. 13. Example 3.9: Nonsymmetric peak and anti-peak interaction of DP equation with the initial condition (52). Periodic boundary condition in $[-25,25] . P^{4}$ elements and a uniform mesh with $N=256$ cells.

434 .

[11] A. Constantin AND J. Escher, Global existence and blow-up for a shallow water equation, Annali della Scuola normale superiore di Pisa. Classe di scienze, 25 (1998), pp. 303-328.

[12] A. Constantin and D. Lannes, The hydrodynamical relevance of the Camassa-Holm and Degasperis-Procesi equations, Archive for Rational Mechanics and Analysis, 192 (2009), pp. $165-186$.

[13] B. CockBurn And S. Y. Lin And C. W. ShU, TVB Runge-Kutta local projection discontinuous Galerkin finite element method for conservation laws III: one dimensional systems, J. Comput. Phys., 84 (1989), pp. 90-113.

[14] B. CockBurn And C. W. ShU, TVB Runge-Kutta local projection discontinuous Galerkin finite element method for conservation laws II: General framework, Math. Comput., 52 (1989), pp. 411-435.

[15] B. Cockburn and S. Hou And C. W. Shu, The Runge-Kutta local projection discontinuous Galerkin finite element method for conservation laws IV: the multidimensional case, Math. Comput., 54 (1990), pp. 545-581.

[16] B. Cockburn And C. W. Shu, The Runge-Kutta discontinuous Galerkin method for conservation laws V: multidimensional systems, J. Comput. Phys., 141 (1998), pp. 199-224.

[17] B. Cockburn and C. W. Shu, The local discontinuous Galerkin methods for time-dependent convection-diffusion systems, SIAM J. Numer. Anal., 35 (1998), pp. 2440-2463.

[18] A. Degasperis, D. Holm, And A. Hone, A new integrable equation with peakon solutions, Theoret. and Math. Phy., 133 (2002), pp. 1463-1474.

[19] A. Degasperis And M. Procesi, Asymptotic integrability, Symmetry and Perturbation Theory. World Sci. Publ., River Edge, NJ, 1999.

[20] J. Escher, Y. LiU, AND Z. YIN, Global weak solutions and blow-up structure for the degasperisprocesi equation, J. Funct. Anal., 241 (2006), pp. 457-485.

[21] J. Escher, Y. LiU, AND Z. Yin, Shock waves and blow-up phenomena for the periodic 


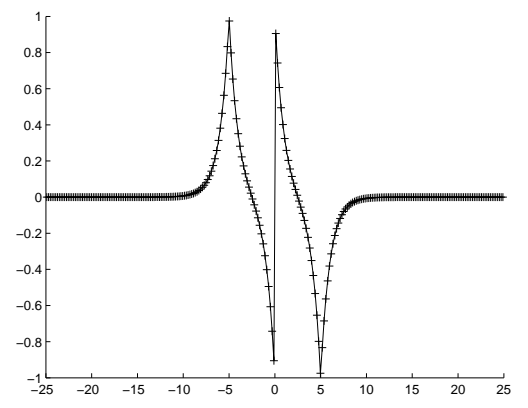

$\mathrm{t}=0$

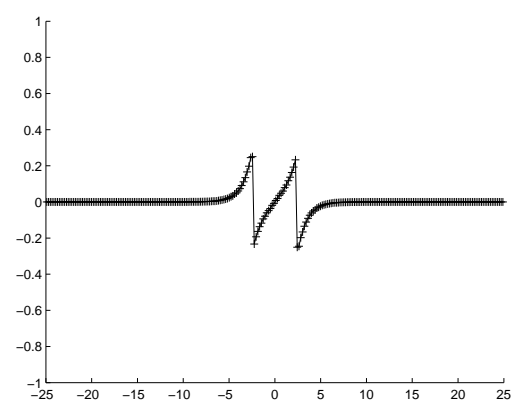

$\mathrm{t}=5.32$

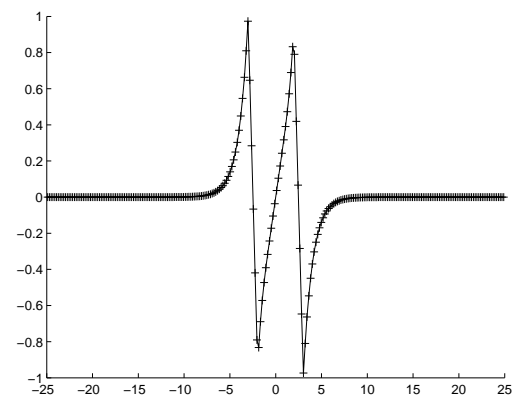

$\mathrm{t}=2$

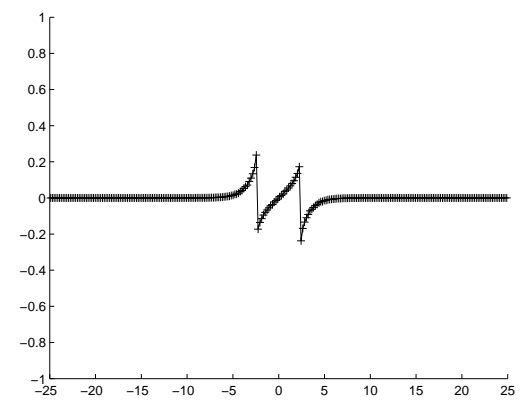

$\mathrm{t}=7$

FIG. 14. Example 3.10: Peakon, shock peakon and anti-peakon interaction of DP equation with the initial condition (53). Periodic boundary condition in $[-25,25] . P^{4}$ elements and a uniform mesh with $N=256$ cells.

Degasperis-Procesi equation, Indiana Univ. Mat. J., 56 (2007), pp. 87-117.

[22] B. Feng and Y. Liu, An operator splitting method for the Degasperis-Procesi equation, J. Comput. Phys., 228 (2009), pp. 7805-7820.

[23] B. Fuchssteiner AND A. S. FoKAS, Symplectic structures, their bäcklund transformations and hereditary symmetries, Physica D, 4 (1981), pp. 47-66.

[24] D. Furihata AND M. Mori, General derivation of finite difference schemes by means of a discrete variation, Trans. Japan Soc. Ind. Appl. Math., 8 (1998), pp. 317-340.

[25] G. Gassner, F. Lörcher, And C. D. Munz, A contribution to the construction of diffusion fluxes for finite volume and discontinuous Galerkin schemes, J. Comput. Phys., 224 (2007), pp. 1049-1063.

[26] T. G. HA AND H. Liu, On traveling wave solutions of the $\theta$-equation of dispersive type, to appear in JMAA.

[27] H. HoEL, A numerical scheme using multi-shockpeakons to compute solutions of the Degasperis-Procesi equation, Electron. J. Differential Equations, 2007 (2007), pp. 1-22.

[28] D. D. Holm ANd M. F. Staley, Wave structures and nonlinear balances in a family of $1+1$ evolutionary PDEs, SIAM J. Appl. Dyn. Syst., 2 (2003), pp. 323-380.

[29] R. S. Johnson, Camassa-Holm, Korteweg-de Vries and related models for water waves, J. Fluid Mech., 455 (2002), pp. 63-82.

[30] B. van Leer and S. Nomura, Discontinuous Galerkin for diffusion, Proceedings of 17th AIAA Computational Fluid Dynamics Conference (June 6 2005), AIAA-2005-5108.

[31] J. LenelLS, Traveling wave solutions of the Degasperis-Procesi equation, J. Math. Anal. Appl., 306 (2005), pp. $72-82$.

[32] H. LiU, Optimal error estimates of the direct discontinuous Galerkin method for convectiondiffusion equations, accepted in Math. Comp. (2013).

[33] H. LiU And J. YAN, local discontinuous Galerkin method for the Korteweg-de Vries equation with boundary effect, J. Comput. Phys., 215 (2006), pp. 197-218. 
[34] H. LiU AND J. YAN, The direct discontinuous Galerkin (DDG) method for diffusion problems, SIAM J. Numer. Anal., 47 (2009), pp. 675-698.

[35] H. LiU AND J. YAN, The direct discontinuous Galerkin (DDG) method for diffusion with interface corrections, Commun. Comput. Phys., 8 (2010), pp. 541-564.

[36] H. LiU AND Z. YIN, Global regularity, and wave breaking phenomena in a class of nonlocal dispersive equations, Contemporary Mathematics, 526 (2011), pp. 274-294.

[37] Y. LiU And Z. Yin, Global existence and blow-up phenomena for the Degasperis-Procesi equation, Commun. Math. Phys., 267 (2006), pp. 801-820.

[38] H. Lundmark, Formation and dynamics of shock waves in the Degasperis-Procesi equation, J. Nonlinear Sci., 17 (2007), pp. 169-198.

[39] H. Lundmark and J. Szmigielski, Multi-peakon solutions of the Degasperis-Procesi equation, Inverse Problems, 19 (2003), pp. 1241-1245.

[40] H. Lundmark and J. Szmigielski, Degasperis-Procesi peakons and the discrete cubic string, IMRP Int. Math. Res. Pap., 2005 (2005), pp. 53-116.

[41] Y. Matsuno, Multi-soliton solutions of the Degasperis-Procesi equation and their peakon limit, Inverse Problems, 21 (2005), pp. 1553-1570.

[42] Y. Matsuno, The n-soliton solution of the Degasperis-Procesi equation, Inverse Problems, 21 (2005), pp. 2085-2101.

[43] T. Matsuo, Dissipative/conservative Galerkin method using discrete partial derivatives for nonlinear evolution equations, J. Comput. Appl. Math., 218 (2008), pp. 506-521.

[44] R. I. McLachlan, G. R. W. Quispel, and N. Robidoux, Geometric integration using discrete gradients, Philosophical Transactions of the Royal Society of London. Series A: Mathematical, Physical and Engineering Sciences, 357 (1999), pp. 1021-1045.

[45] Y. Miyatake and T. Matsuo, Conservative finite difference schemes for the DegasperisProcesi equation, J. Comput. Appl. Math., 236 (2012), pp. 3728-3740.

[46] W. ReEd AND T. HiLl, Triangular mesh methods for the neutrontransport equation, La-ur-73479, Los Alamos Scientific Laboratory, 1973.

[47] Y. Xu AND C. W. Shu, Local discontinuous Galerkin methods for the Degasperis-Procesi equation, Comm. Comput. Phys., 10 (2011), pp. 474-508.

[48] Y. XU And C. W. Shu, Local discontinuous Galerkin methods for two classes of twodimensional nonlinear wave equations, Physica D, 208 (2005), pp. 21-58.

[49] Y. XU AND C. W. Shu, Error estimates of the semi-discrete local discontinuous Galerkin method for nonlinear convection-diffusion and KdV equations, Comput. Methods Appl. Mech. Engrg., 196 (2007), pp. 3805-3822.

[50] Y. Xu AND C. W. Shu, A local discontinuous Galerkin method for the Camassa-Holm equation, SIAM J. Numer. Anal., 46 (2008), pp. 1998-2021.

[51] Y. XU And C. W. Shu, Local discontinuous Galerkin method for higher order time-dependent partial differential equations, Commun. Comput. Phys., 7 (2010), pp. 1-46.

[52] J. YAn AND C. W. Shu, A local discontinuous Galerkin method for KdV type equations, SIAM J. Numer. Anal., 40 (2003), pp. 769-791.

[53] N. Yi, Y. HuAnG, AND H. LiU, A direct discontinuous Galerkin method for the generalized Korteweg-de Vries equation: Energy conservation and boundary effect, J. Comput. Phys., 242 (2013), pp. 351-366.

[54] Z. YIN, Global existence for a new periodic integrable equation, J. Math. Anal. Appl., 283 (2003), pp. 129-139.

[55] Z. YIN, On the Cauchy problem for an integrable equation with peakon solutions, Illinois J. Math., 47 (2003), pp. 649-666.

[56] Z. YIN, Global weak solutions for a new periodic integrable equation with peakon solutions, J. Funct. Anal., 212 (2004), pp. 182-194. 
H. LIU, Y. HUANG, AND N. YI 